\title{
Two-year Efficacy and Safety of Subcutaneous Tocilizumab in Combination with Disease-modifying Antirheumatic Drugs Including Escalation to Weekly Dosing in Rheumatoid Arthritis
}

\author{
Alan Kivitz, Ewa Olech, Michael A. Borofsky, Beatriz Zazueta, Federico Navarro-Sarabia, \\ Sebastião C. Radominski, Joan T. Merrill, César Pacheco-Tena, Jinglan Pei, \\ Clare Nasmyth-Miller, and Janet E. Pope
}

ABSTRACT. Objective. To evaluate the longterm efficacy and safety of subcutaneous tocilizumab (TCZ-SC) every 2 weeks ( 2 w) over 2 years in patients with rheumatoid arthritis who have an inadequate response to disease-modifying antirheumatic drugs (DMARD).

Methods. Patients $(\mathrm{n}=656)$ were randomized 2:1 to TCZ-SC $162 \mathrm{mg}$ q2w or placebo-SC q2w plus DMARD. After a 24-week double-blind period, patients $(n=457)$ were rerandomized to open-label TCZ-SC q2 $\mathrm{w}$ by means of prefilled syringe or autoinjector. Escape therapy with weekly TCZ-SC was available for patients with inadequate efficacy from Week 12. Maintenance of response and safety to 2 years was assessed. Analyses used nonresponder imputation.

Results. The American College of Rheumatology (ACR) 20 response after TCZ-SC was maintained beyond Week 24 and was $>70 \%$ at each timepoint. ACR50/70, 28-joint Disease Activity Score remission, and $\geq 0.30$ decrease from baseline in the Health Assessment Questionnaire-Disability Index response rates were also maintained after Week 24 in the TCZ-SC arm $(\geq 50 \%,>25 \%,>32 \%$ and $>56 \%$, respectively). Following escape for inadequate efficacy, many patients achieved ACR20 at the end of the study, $35 \%$ after escape from TCZ-SC, and $63 \%$ from placebo. The rates of serious adverse events [(11.20/100 patient-years (PY)] including serious infections (3.25/100 PY) were stable through Week 96. No association between anti-TCZ antibody development and loss of efficacy or adverse events was observed.

Conclusion. Efficacy and safety of TCZ-SC q2w was maintained up to 2 years and remained comparable with previously published data for intravenous TCZ. Dose escalation to weekly TCZ-SC was associated with ACR responses in prior nonresponders and was well tolerated. (First Release December 15 2017; J Rheumatol 2018;45:456-64; doi:10.3899/jrheum.161539)

Key Indexing Terms:

RHEUMATOID ARTHRITIS

BIOLOGICAL THERAPY

CLINICAL TRIAL

From the Altoona Center for Clinical Research, Duncansville, Pennsylvania; University of Nevada School of Medicine, Las Vegas, Nevada; Clinical Research Center of Reading, Reading, Pennsylvania; Oklahoma Medical Research Foundation, Oklahoma City, Oklahoma; Genentech, South San Francisco, California, USA; Universidad Autónoma de Chihuahua, Chihuahua; Centro de Investigacion en Enfermedades Reumaticas, Mexicali, Baja California, Mexico; Hospital Virgen Macarena, Servicio de Reumatología, Sevilla, Spain; Universidade Federal do Paraná, Curitiba, Brazil; Roche Pharmaceuticals, Welwyn Garden City, London, UK; St. Joseph's Health Centre, Western University, London, Ontario, Canada.

This study was funded by Genentech, a member of the Roche Group, and Hoffmann-La Roche. Genentech and Hoffmann-La Roche sponsored the study and participated in the design of the study, as well as in the collection, analysis, and interpretation of the data. A. Kivitz received consulting fees and speakers bureau fees from Genentech. E. Olech received research grants and consulting fees from Genentech.

F. Navarro-Sarabia received consulting fees and speakers bureau fees from Roche Pharmaceuticals. S.C. Radominski received research grants from Roche Pharmaceuticals. J.T. Merrill received consulting fees from Roche Pharmaceuticals and Genentech.J. Pei is an employee of Genentech. C. Nasmyth-Miller is an employee of Roche Pharmaceuticals.
A. Kivitz, MD, Altoona Center for Clinical Research; E. Olech, MD, University of Nevada School of Medicine; M.A. Borofsky, MD, Clinical Research Center of Reading; B. Zazueta, MD, Centro de Investigacion en Enfermedades Reumaticas; F. Navarro-Sarabia, MD, Hospital Virgen Macarena, Servicio de Reumatología; S.C. Radominski, MD, Universidade Federal do Paraná; J.T. Merrill, MD, Oklahoma Medical Research Foundation; C. Pacheco-Tena, MD, Universidad Autónoma de Chihuahua; J.Pei, BS, Genentech; C. Nasmyth-Miller, DPhil, Roche Pharmaceuticals; J.E. Pope, MD, St. Joseph's Health Centre, Western University.

Address correspondence to Dr. A. Kivitz, Altoona Arthritis and Osteoporosis Center, 175 Meadowbrook Lane, PO Box 909, Duncansville, Pennsylvania 16635,USA.E-mail:ajkivitz@yahoo.com

Full Release Article. For details see Reprints and Permissions at jrheum.org Accepted for publication September 19, 2017.

Tocilizumab (TCZ) is a recombinant humanized monoclonal antibody targeted against human interleukin 6 receptor- $\alpha$, and subcutaneous TCZ (TCZ-SC) was developed as an alternative to intravenous TCZ (TCZ-IV). TCZ-IV has well-estab- 
lished efficacy and a manageable safety profile, both as monotherapy or in combination with disease-modifying antirheumatic drugs (DMARD), in patients with rheumatoid arthritis (RA) ${ }^{1,2,3,4}$. The safety and efficacy of TCZ-SC are comparable with TCZ-IV as monotherapy and with DMARD, and TCZ-SC has been approved for use globally $5,6,7,8,9$.

At 2 years, the phase III TCZ-SC trials SUMMACTA ${ }^{8}$ [weekly (qw) with DMARD)] and MUSASHI ${ }^{9}$ [every 2 weeks ( $\mathrm{q} 2 \mathrm{w})$ as monotherapy] showed that TCZ-SC maintained efficacy and safety comparable to TCZ-IV. The phase III BREVACTA study evaluated the efficacy and safety of biweekly TCZ-SC in combination with DMARD in patients with moderate to severe RA and an inadequate response to DMARD ${ }^{6}$. TCZ-SC $162 \mathrm{mg}$ q2w demonstrated significantly greater efficacy, including inhibiting joint damage, versus placebo (PBO)-SC at Week 24. TCZ-SC q2w was well tolerated and its safety profile was consistent with the TCZ-IV profile, except for increased injection-site reactions (ISR) ${ }^{6}$.

Our analysis evaluated the longterm efficacy and safety of TCZ-SC q2w up to Week 96. Data for patients who received TCZ qw following escalation from either TCZ-SC q2w or PBO q2w to Week 96 are included.

\section{MATERIALS AND METHODS}

Participants. Patients signed informed consent documents approved by an institutional review board or independent ethics committee. Our study was conducted in accordance with the Declaration of Helsinki and Good Clinical Practice. Quorum Review IRB approved the study (25643/2) and approval was obtained for each study site (about 170 centers). Patient selection criteria were previously described ${ }^{6}$. All patients had an inadequate response to $\geq 1$ DMARD, and $21 \%$ had an inadequate response to anti-tumor necrosis factor (anti-TNF) agents. Patients continued to receive $\geq 1$ nonbiologic DMARD at the stable pre-entry dose.

Study design. BREVACTA (clinicaltrials.gov, identifier: NCT01232569) was a phase III, randomized, double-blind, 2-arm, 2-year PBO-controlled, parallel-arm multicenter trial with a 24 -week double-blind period, followed by a 72 -week open-label extension period (Supplementary Figure 1, available with the online version of this article). At baseline, patients were randomized 2:1 to receive TCZ-SC $162 \mathrm{mg} \mathrm{q} 2 \mathrm{w}$ or PBO-SC q2w by prefilled syringe. At Week 24, patients were rerandomized 1:1 to receive TCZ-SC $162 \mathrm{mg} \mathrm{q} 2 \mathrm{w}$ by prefilled syringe or autoinjector. Escape therapy with TCZ-SC qw was available from weeks 12 to 48 to patients with $<20 \%$ improvement in swollen joint count (SJC) and tender joint count (TJC) from baseline. After Week 48, patients could receive TCZ-SC $162 \mathrm{mg}$ qw if there was $\leq 70 \%$ improvement from baseline in SJC and TJC.

Outcomes and assessments. Evaluations included the percentage of patients who achieved $20 \%, 50 \%$, and $70 \%$ improvements in response per the American College of Rheumatology criteria (ACR20/50/70); remission based on the Disease Activity Score using 28 joints calculated with erythrocyte sedimentation rate (DAS28 < 2.6); and decreases in Health Assessment Questionnaire-Disability Index (HAQ-DI) scores of $\geq 0.30$. Radiographic progression from baseline to weeks 24 and 48 was assessed using the modified total Sharp score analysis (mTSS) ${ }^{10}$ and performed by 2 primary readers, with an adjudicator involved for any discrepancies. Safety assessments included adverse events (AE), laboratory measurements, and immunogenicity ${ }^{11}$.

Statistical analysis. Data from the prefilled syringe and autoinjector devices were combined. The efficacy and laboratory analyses used the inten- tion-to-treat (ITT) population: 1 group received TCZ-SC continuously (TCZ-SC arm), and 1 group received PBO to Week 24 and was then rerandomized to TCZ-SC (PBO-TCZ-SC arm). The nonresponder analysis included all patients who completed Week 24 , were rerandomized, received $\geq 1$ dose of rerandomized study treatment, and had a valid assessment at the timepoint analyzed. No formal statistical analyses were performed at Week 96. For completer analysis, the efficacy data were reported as observed in patients who completed the study, with no imputation of missing data. Radiographic data were evaluated at baseline, Week 24, and Week 48, with early withdrawal or escape therapy readings evaluated up to the Week 48 visit. Missing Week 48 data were imputed using linear extrapolation.

The safety population consisted of 3 groups: TCZ-SC q2w (safety events in the double-blind and open-label periods), PBO-TCZ-SC (events from TCZ-SC in the open-label period), and PBO (events in the double-blind period). All patients had $\geq 1$ postdose assessment of safety. In the PBOTCZ-SC arm, AE in patients who withdrew, died, or escaped in the first 24 weeks were not included. For patients who continuously received TCZ-SC in the double-blind and open-label periods, all events were included. The ITT pharmacokinetic population consisted of patients who provided $\geq 1$ pharmacokinetic sample for TCZ and serum interleukin 6 receptor in the double-blind or open-label periods.

Efficacy and safety analyses for the escape population included all patients who received TCZ-SC qw. Only data from the time of escape were included in the escape patient analyses. Patients were rebaselined, and the last assessment prior to their first qw dose was considered the baseline. All analyses were exploratory.

\section{RESULTS}

Patient disposition and baseline characteristics. Of the 461 patients who completed Week 24 without escape therapy, 457 continued in this longterm extension study; 338 were rerandomized from blinded TCZ-SC to receive open-label TCZSC q2w, and 119 were rerandomized from blinded PBO to open-label TCZ-SC q2w (Figure 1). During the open-label period, 43 patients (12.7\%) who initiated TCZ-SC and 11 (9.2\%) who initiated PBO withdrew. The most common reasons for withdrawal were patient decision (1 patient withdrew because of pregnancy; $n=21)$ and $\mathrm{AE}(\mathrm{n}=19)$.

Of the 189 escape patients (TCZ-SC qw), 162 received escape therapy prior to Week 24 . There were 14 patients who withdrew from the escape-TCZ-SC group and 17 from the escape-PBO group after Week 24; the most common reason was AE (6 patients/arm).

Patient demographics and clinical characteristics were balanced across the treatment groups (Supplementary Table 1 , available with the online version of this article).

Efficacy. In nonresponder analyses, ACR20 responses in the TCZ-SC group were maintained from $78 \%$ at Week 24 to $72 \%$ at Week 96; in the PBO-TCZ-SC group, the ACR20 response increased from 56\% at Week 24 to $81 \%$ at Week 36 and was sustained thereafter through Week 96 (Figures 2A and 2B). From weeks 24 to 96, DAS28 remission rates increased and the rates for a decrease in HAQ-DI scores of $\geq 0.30$ were sustained, respectively, in the TCZ-SC group (DAS28: $33 \%$ and 45\%; HAQ-DI: $59 \%$ and 57\%; Figures $2 \mathrm{C}$ and 2D). A greater percentage of patients in the PBOTCZ-SC group achieved DAS28 remission or a decrease in HAQ-DI score of $\geq 0.3$ from weeks 24 to 96 , respectively

Personal non-commercial use only. The Journal of Rheumatology Copyright @ 2018 . All rights reserved. 


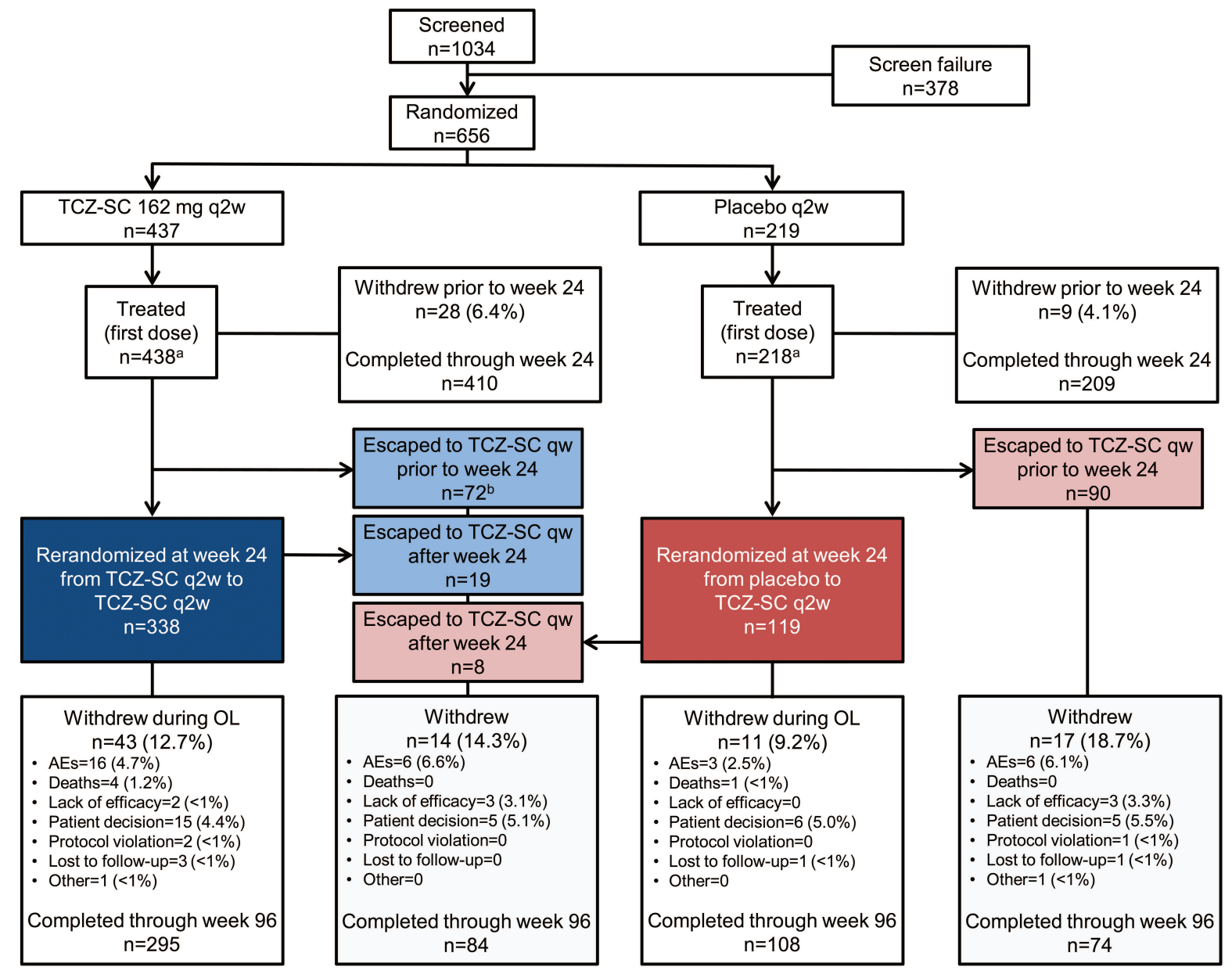

Figure 1. Patient disposition over 96 weeks. One patient treated with TCZ-SC had no postbaseline data and was excluded from the safety population. At Week 24, patients in each arm were rerandomized 1:1 to receive TCZ-SC using either an autoinjector or a prefilled syringe during the OL period. However, for the analysis, patients were grouped into those who received TCZ-SC continuously (regardless of device used) and those who were rerandomized from PBO to TCZ-SC at Week 24. ${ }^{\text {a }}$ One patient from the PBO group received TCZ at baseline. ${ }^{\mathrm{b}}$ One patient who escaped in the TCZ q2w arm was analyzed in the escape PBO to TCZ-SC qw group because this patient received PBO prior to receiving escape therapy with TCZ qw. AE: adverse event; OL: open-label; q2w: every 2 weeks; qw: weekly; TCZ-SC: subcutaneous tocilizumab; PBO: placebo.

(DAS28: 4\% and 42\%; HAQ-DI: 47\% and 57\%; Supplementary Figure 2, available with the online version of this article). Similar efficacy results were observed by completer analysis (Supplementary Figures 2 and 3, available with the online version of this article).

Patients in the PBO-TCZ-SC group experienced more radiographic progression at Week 48 from baseline than patients in the TCZ-SC group (mean mTSS change: 1.48 vs 0.64; Figure 3A; cumulative distribution plot in Figure 3B). The mean annualized progression rate for mTSS decreased in both treatment arms through Week 48 and was 2-fold higher in the PBO-TCZ-SC arm than in the TCZ-SC arm (0.57 vs 0.29 ).

Efficacy by weight stratification. Evidence of efficacy, as measured by ACR20/50/70, was demonstrated across all weight groups ( $<60 \mathrm{~kg}, 60$ to $<100 \mathrm{~kg}, \geq 100 \mathrm{~kg}$ ) with both TCZ-SC and PBO-TCZ-SC arms through to Week 96.
However, response rates were variable, and the analysis was limited by the small number of patients in some groups (Supplementary Figure 4, available with the online version of this article).

Pharmacokinetics. The mean TCZ concentration was stable in all patients from weeks 48 to 96 (Supplementary Figure 5, available with the online version of this article). In patients who received PBO up to Week 24, after rerandomization the mean pre-dose TCZ concentrations increased.

Safety. The safety profile of TCZ-SC q2w over 96 weeks was consistent with that in the double-blind period (Table 1; Supplementary Figure 6, available with the online version of this article). The rate of AE/100 PY for TCZ-SC q2w did not increase from weeks 24 to 96 , and the rate of serious $\mathrm{AE}$ (SAE) was low and consistent over time (Supplementary Figure 6). The rate of withdrawals/100 PY because of AE for TCZ-SC q2w was 4.22, and 1.30 for the PBO to TCZ-SC 
A

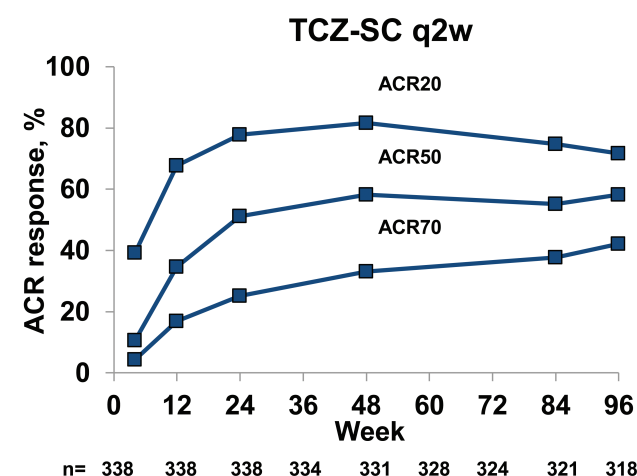

B
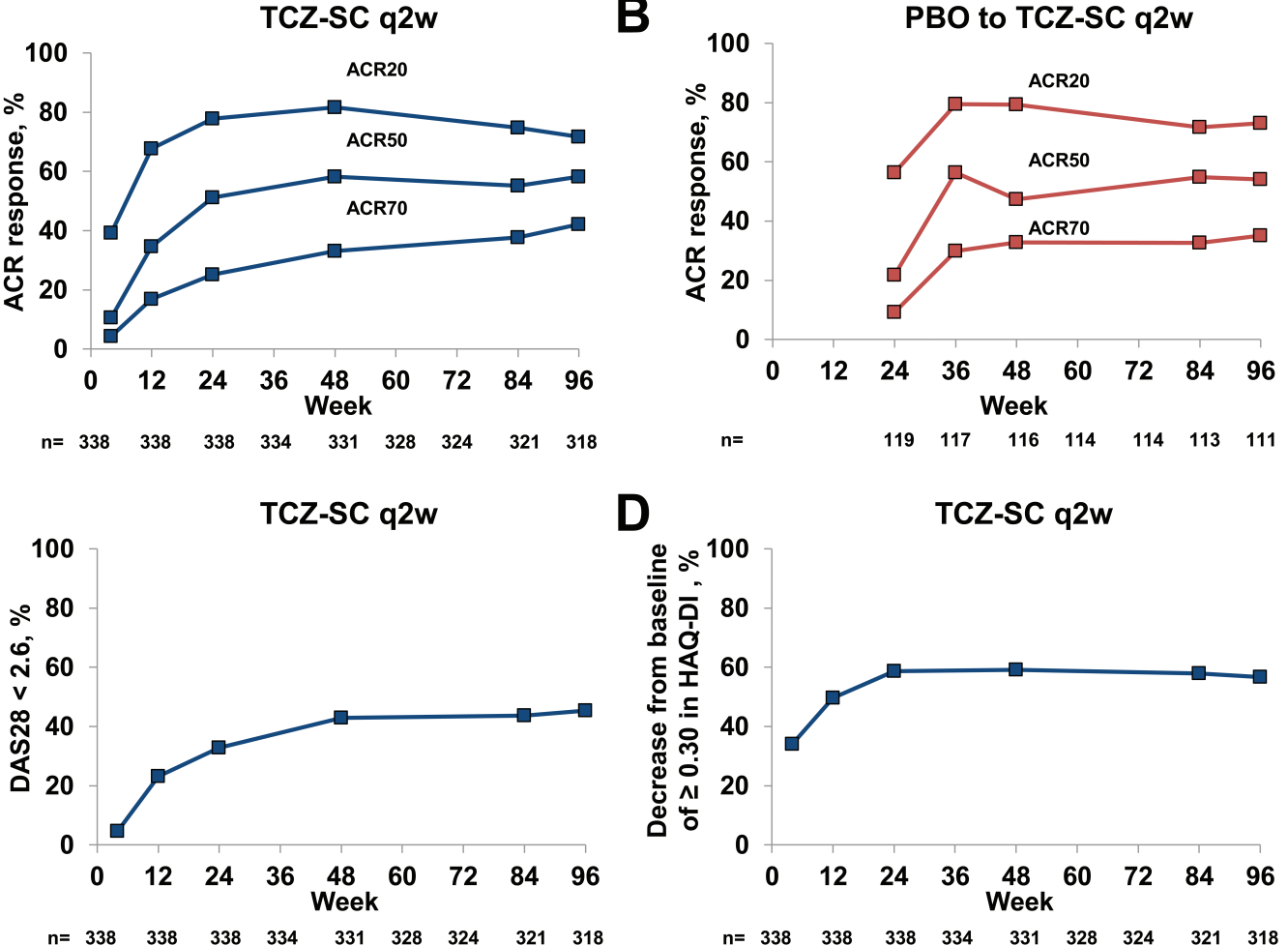

D

TCZ-SC q2w

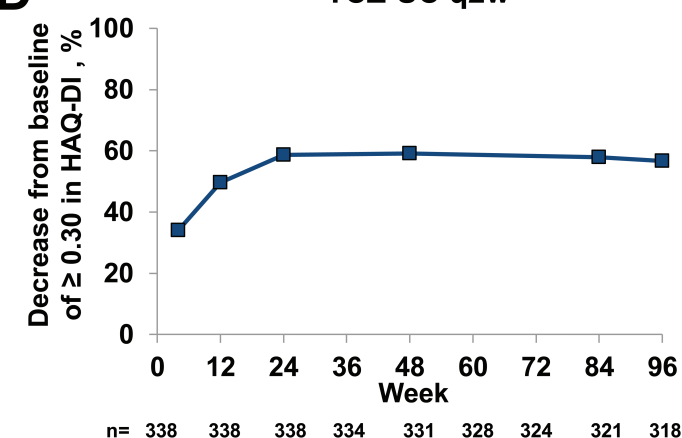

Figure 2. Nonresponder analysis of the randomized populations: percentage of patients who achieved $20 \%, 50 \%$, and $70 \%$ improvements in response per the ACR20/ACR50/ACR70 criteria who (A) received TCZ-SC continuously, and (B) were rerandomized from placebo to TCZ-SC (PBO-TCZ-SC) at Week 24. Percentage of patients who received TCZ-SC continuously and who achieved (C) remission based on DAS28 $<2.6$, and (D) decreases in HAQ-DI scores of $\geq 0.30$ from baseline over 96 weeks (intention-to-treat population). The $\mathrm{n}$ values refer to the nonresponder analysis. ACR: American College of Rheumatology; DAS28: 28-joint Disease Activity Score; HAQ-DI: Health Assessment Questionnaire-Disability Index; PBO: placebo; q2w: every 2 weeks; TCZ-SC: subcutaneous tocilizumab.

q2w group (Supplementary Figure 6). For TCZ-SC q2w, infections were the most common AE and SAE (Table 1). The rate of serious infections/100 PY for TCZ-SC q2w was 3.25, and was 0.65 for the PBO to TCZ-SC q2w group. Rates of serious infections/100 PY did not increase with increasing exposure to treatment (Supplementary Figure 6). In the TCZ-SC group, 16 patients developed 20 serious infections: the most common were pneumonia, sepsis, lower respiratory tract, bacterial arthritis, osteomyelitis, and pulmonary tuberculosis (2 patients each). One patient in the PBO-TCZ-SC group experienced a serious infection (urinary tract). Opportunistic infections during the open-label period were reported in 2 patients (esophageal candidiasis and atypical pneumonia) in the TCZ-SC group; 1 patient had coccidioidomycosis during the double-blind period as previously reported (Supplementary Table 2, available with the online version of this article). No opportunistic infections were reported during the open-label period in the PBO-TCZ-SC group.

There were 6 malignancies that occurred over the 2-year study (Table 1), 3 of which were reported in 3 patients during the open-label period in the TCZ-SC group: rectal cancer, thyroid neoplasm, and ovarian cancer. One patient in the PBO-TCZ-SC group developed 2 malignancies: grade 2 squamous cell carcinoma and grade 3 non-Hodgkin lymphoma.

There were 9 deaths that occurred during the entire study, 5 of which occurred during the open-label period (Supplementary Table 3, available with the online version of this article). The rate of death in the TCZ-SC group during the double-blind and open-label periods was 1.14/100 PY (95\% CI 0.46-2.34); in the PBO-TCZ-SC group, it was 0.65/100 PY (95\% CI 0.02-3.61) during the open-label period only. No deaths occurred in the PBO-TCZ-SC group during the double-blind period. The most common causes of death over 96 weeks were cardiovascular events (myocardial infarction and angina, $\mathrm{n}=4$ ) and infections (sepsis and lower respiratory tract infection, $\mathrm{n}=3$ ). One patient who was hospitalized and treated with antibiotics for fever of unknown origin developed multiple organ failure and died. One death is described in the escape section.

The rates of hypersensitivity AE (defined as all AE except ISR) that occurred during or within $24 \mathrm{~h}$ of injection and that

$$
\text { Personal non-commercial use only. The Journal of Rheumatology Copyright (C) 2018. All rights reserved. }
$$



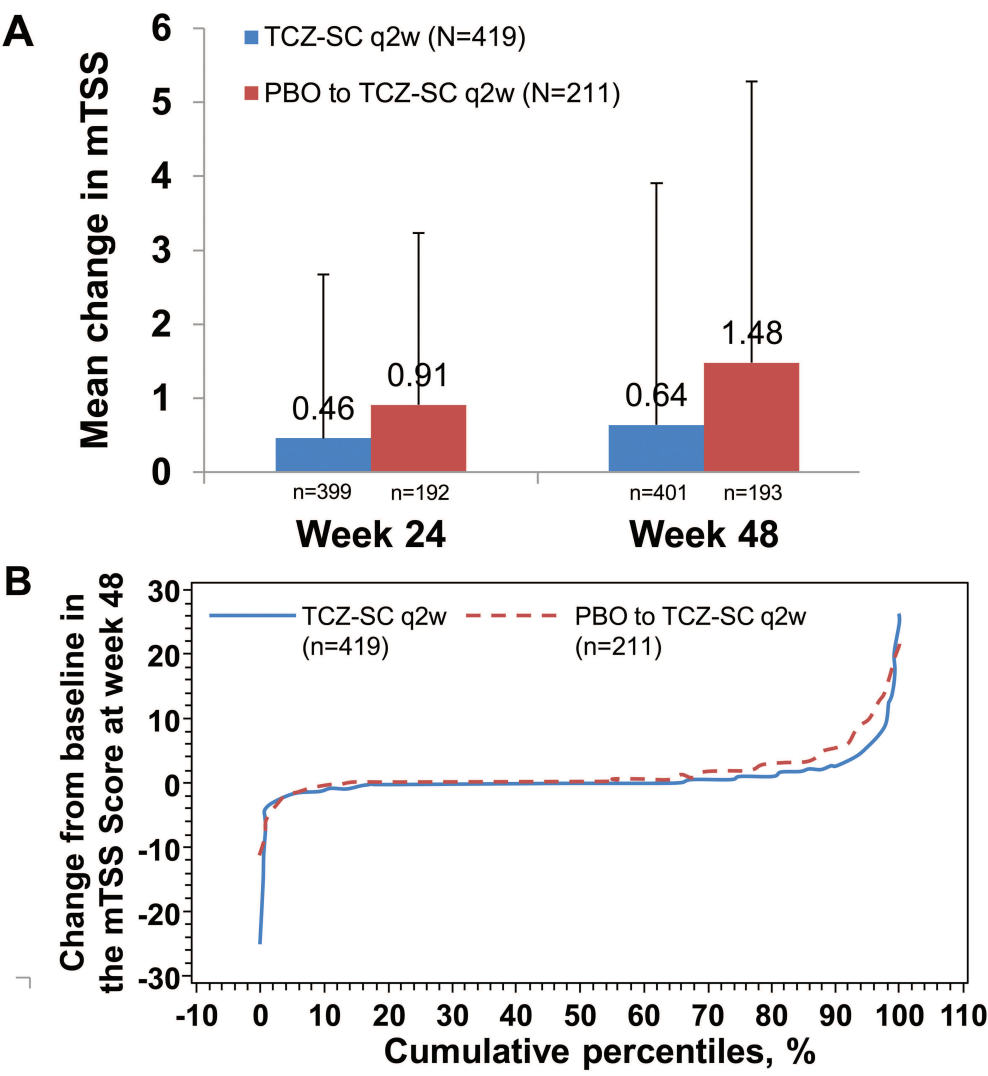

Figure 3. Radiographic results for pooled treatment arms as randomized at baseline: TCZ-SC q2 $\mathrm{w}$ and $\mathrm{PBO}$ from weeks 24 to 48. Patients randomized at baseline to receive $\mathrm{PBO}$ were rerandomized at Week 24 to receive TCZ-SC q2w. Missing Week 48 data were imputed using linear extrapolation. Patient data were extrapolated if they had a baseline assessment and $\geq 1$ postbaseline assessment. Escape and withdrawal patients were extrapolated from the time they withdrew or escaped. A 30-day window was allowed for valid assessment. (A) Mean change in mTSS from baseline to weeks 24 and 48. (B) Cumulative distribution plot of mTSS change. PBO: placebo; mTSS: modified total Sharp score; $\mathrm{q} 2 \mathrm{w}$ : every 2 weeks; TCZ-SC: subcutaneous tocilizumab.

A

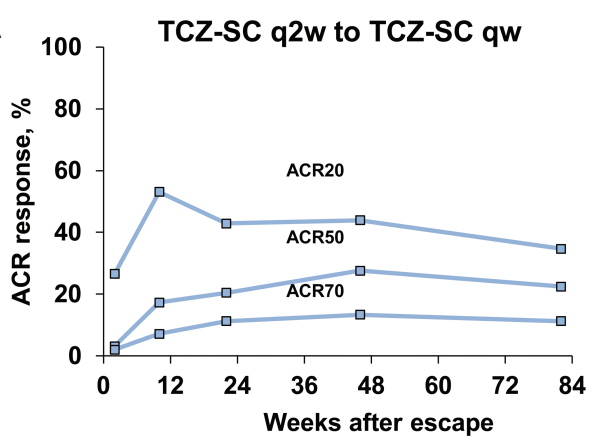

B

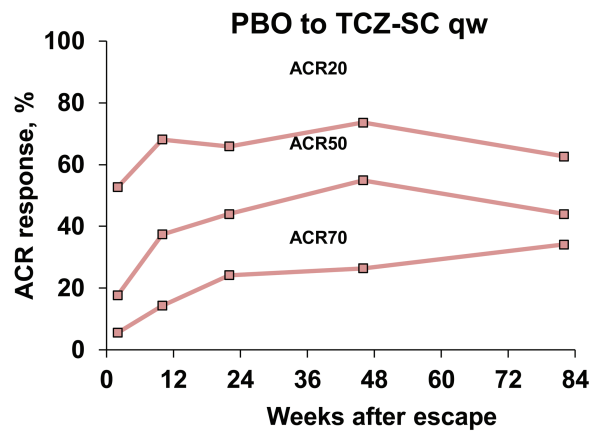

98

C

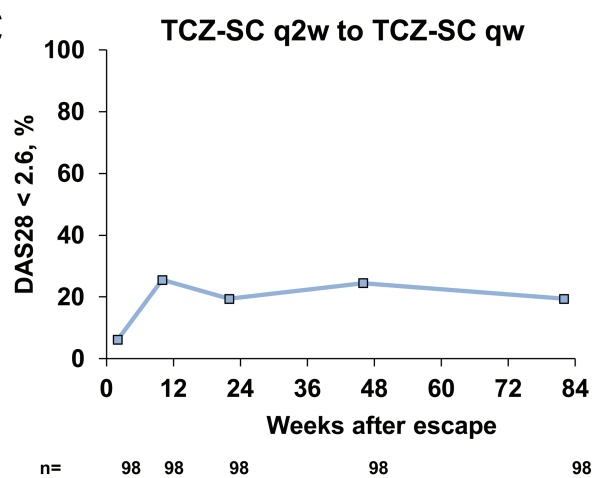

91

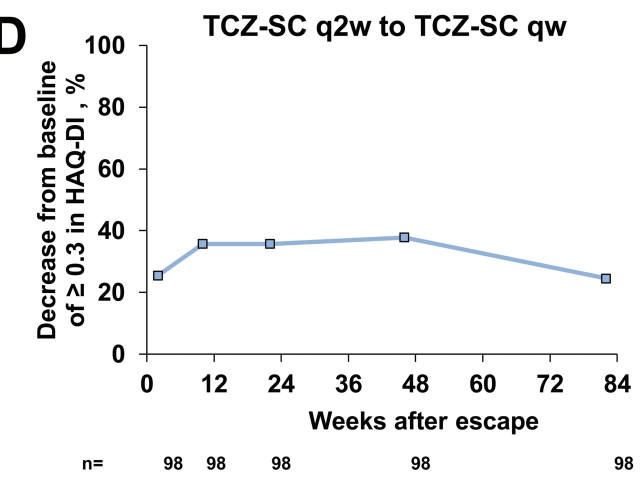

Figure 4. Nonresponder analysis of the escape populations: percentage of patients in the (A) escape-TCZ-SC $\mathrm{q} 2 \mathrm{w}$ group and (B) escape-PBO group who received escape therapy weekly and achieved 20\%, 50\%, and $70 \%$ improvements in response per the American College of Rheumatology criteria (ACR20/ACR50/ACR70). Percentage of patients in the escapeTCZ-SC q2w group who received escape therapy qw and achieved (C) remission based on DAS2 $8<2.6$, and (D) decreases in HAQ-DI scores of $\geq 0.30$ (escape population, as observed). For the escape population, patients were rebaselined at escape. Data were collected from baseline (last assessment prior to the first dose of escape therapy) up to Week 96 (for a maximum of 84 weeks after escape). The $\mathrm{n}$ values refer to the nonresponder analysis. DAS28: 28-joint Disease Activity Score; HAQ-DI: Health Assessment Questionnaire-Disability Index; PBO: placebo; qw: weekly; q2w: every 2 weeks; TCZ-SC: subcutaneous tocilizumab. 
Table 1. Overview of cumulative AE and SAE through Week $96^{\mathrm{a}, \mathrm{b}}$. Values are rate/100 PY (95\% CI), unless otherwise specified.

\begin{tabular}{|c|c|c|c|}
\hline Variables & TCZ-SC q2 $w^{\mathrm{c}}, \mathrm{n}=437$ & PBO to TCZ-SC ${ }^{d}$ q2w, $n=120$ & $\mathrm{PBO}^{\mathrm{e}}, \mathrm{n}=218$ \\
\hline PY exposure & 615.95 & 154.20 & 82.80 \\
\hline $\mathrm{AE}$ & $332.82(318.57-347.55)$ & $240.60(216.74-266.37)$ & $353.86(314.50-396.79)$ \\
\hline SAE & $11.20(8.72-14.18)$ & $1.30(0.16-4.69)$ & $14.49(7.49-25.32)$ \\
\hline No. events & 69 & 2 & 12 \\
\hline Withdrawals because of $\mathrm{AE}$ & $4.22(2.76-6.18)$ & $1.30(0.16-4.69)$ & $4.83(1.32-12.37)$ \\
\hline No. events & 533 & 115 & 81 \\
\hline Serious infections & $3.25(1.98-5.01)$ & $0.65(0.02-3.61)$ & $6.04(1.96-14.09)$ \\
\hline No. events & 20 & 1 & 5 \\
\hline Injection-site reactions & $19.81(16.45-23.65)$ & $1.95(0.40-5.69)$ & $10.87(4.97-20.63)$ \\
\hline No. events & 122 & 3 & 9 \\
\hline Hypersensitivity events ${ }^{\mathrm{f}}$ & $5.20(3.55-7.33)$ & $2.59(0.71-6.64)$ & $14.49(7.49-25.32)$ \\
\hline No. events & 6 & 2 & \\
\hline Serious myocardial infarction & $0.32(0.04-1.17)$ & $0.65(0.02-3.61)$ & 0 \\
\hline No. events & 2 & 1 & \\
\hline Serious bleeding events & $0.16(0.00-0.90)$ & 0 & 0 \\
\hline No. events & 1 & & \\
\hline Deaths & $1.14(0.46-2.34)$ & $0.65(0.02-3.61)$ & 0 \\
\hline No. events & 7 & 1 & \\
\hline
\end{tabular}

${ }^{a}$ No cases of anaphylaxis, serious demyelinating events, serious GI perforations (medically confirmed GI), or serious hepatic events were reported. ${ }^{b}$ Escape patients were excluded from the analysis beginning at the time of escape. ${ }^{\mathrm{C} T C Z-S C ~ q 2 w: ~ p a t i e n t s ~ r e c e i v e d ~ T C Z-S C ~ t h r o u g h o u t ~ t h e ~ s t u d y ; ~ i n c l u d e s ~ a l l ~ s a f e t y ~}$ events from this group in the double-blind and open-label periods. ${ }^{\mathrm{d}} \mathrm{PBO}-\mathrm{TCZ}-\mathrm{SC}$ : patients received PBO and were then rerandomized to TCZ at Week 24 ; includes events from the start of TCZ-SC administration in the open-label period only. ${ }^{\mathrm{e} B O}$ : patients received PBO; includes all events that occurred on PBO in the double-blind period only. ${ }^{\mathrm{f} H y p e r s e n s i t i v i t y ~ e v e n t s ~ d e f i n e d ~ a s ~ a l l ~} \mathrm{AE}$ (except for injection-site reactions) that occurred during or within $24 \mathrm{~h}$ of injection and that were not judged "unrelated" to treatment by the investigator, regardless of whether they were consistent with hypersensitivity. ${ }^{\mathrm{g}}$ Serious hypersensitivity events were defined as all hypersensitivity events that were reported as "serious" hypersensitivity. ${ }^{\text {h}}$ All malignant and unspecified tumors, including nonmelanoma skin cancer AE, were included. AE: adverse events; GI: gastrointestinal; PBO: placebo; PY: patient-years; q2w: every 2 weeks; SAE: serious adverse event; TCZ-SC: subcutaneous tocilizumab.

were not judged "unrelated" to treatment by the investigator, regardless of whether they were clinically consistent with hypersensitivity, were 5.20/100 PY in the TCZ-SC group and 2.59/100 PY in the PBO-TCZ-SC group (Table 1). One patient in the TCZ-SC group had a grade 1 hypersensitivity $\mathrm{AE}$ that led to treatment withdrawal; it manifested as hypotension, mild headache, and dizziness. One patient in the TCZ-SC group had a serious hypersensitivity event (Ludwig angina), but it was not clinically consistent with hypersensitivity. No patients in the PBO-TCZ-SC group reported hypersensitivity $\mathrm{AE}$ that led to study withdrawal or serious hypersensitivity AE. No cases of anaphylaxis, serious demyelinating events, serious gastrointestinal (GI) perforations (medically confirmed), or serious hepatic events occurred in either group.

Throughout the 96 weeks, 39 patients (8.9\%) in the TCZ-SC group experienced 122 ISR and 3 patients $(2.5 \%)$ in the PBO-TCZ-SC group experienced 3 ISR. Most ISR were grade 1 , and none led to withdrawal. The most commonly reported ISR were injection-site erythema and pain.
Laboratory analysis. There were $18(5.3 \%)$ and 11 patients $(3.3 \%)$ in the TCZ-SC group who developed elevated [>3 $\times$ upper limit of normal (ULN)] alanine aminotransferase (ALT) and aspartate aminotransferase (AST) levels, respectively. In the PBO-TCZ-SC group, elevated ALT and AST levels were identified in $8(6.7 \%)$ and 4 patients (3.3\%), respectively (Supplementary Table 4, available with the online version of this article). No patients met the Hy law criteria. Two patients in the TCZ-SC group withdrew because of abnormal ALT levels. Two patients in the PBO-TCZ-SC group and 12 patients in the TCZ-SC group had a dose modification because of abnormal ALT or AST levels.

Grade 3 or 4 neutropenia was identified in 17 patients $(5.0 \%)$ in the TCZ-SC group and $7(5.9 \%)$ in the PBOTCZ-SC group. No patients in the PBO-TCZ-SC group and 4 patients who received TCZ-SC continuously withdrew because of neutropenia. No serious infections were associated with neutropenia. One patient had grade 3 thrombocytopenia. No serious bleeding events were associated with thrombocytopenia.

$$
\text { Personal non-commercial use only. The Journal of Rheumatology Copyright (C) 2018. All rights reserved. }
$$


Patients in the PBO arm who initiated TCZ-SC at Week 24 developed shifts from baseline in lipid variables similar to those in patients who received TCZ-SC and were rerandomized at Week 24 (Supplementary Table 4, available with the online version of this article). Of the patients whose cholesterol was within normal limits at baseline, postbaseline shifts from $\leq 200 \mathrm{mg} / \mathrm{dl}$ to worse values $\geq 240 \mathrm{mg} / \mathrm{dl}$ were observed in $8.0 \%$ of patients. Similar results were observed for low-density lipoprotein cholesterol.

Immunogenicity. There were 9 patients in the TCZ-SC group and 1 patient in the PBO-TCZ-SC group who developed anti-TCZ antibodies postbaseline with both the confirmation and neutralizing assays. No patient who developed anti-TCZ antibodies had hypersensitivity events that led to study withdrawal, anaphylaxis, or ISR, or experienced loss of efficacy (defined as withdrawal owing to lack of efficacy and a pre-withdrawal ACR50 response or European League Against Rheumatism good response).

Safety subgroup analyses by weight. There was considerable variability in AE and SAE rates by weight (Supplementary Table 5, available with the online version of this article).
Efficacy in escape population. In nonresponder analyses, ACR responses in both the escape-PBO and escape-TCZ-SC groups increased, following escalation to TCZ-SC qw (Figures 4A and B). A total of $41 \%$ of patients in the escape-PBO group and 19\% of patients in the escapeTCZ-SC group achieved DAS28 remission at Week 84 (Figure 4C; Supplementary Figure 7A, available with the online version of this article). Similar patterns were observed for other efficacy variables: DAS28 and HAQ-DI scores, and the percentage of patients who achieved a decrease in HAQ-DI $\geq 0.3$ (Figure 4; Supplementary Figure 7). Completer analysis gave similar efficacy results (Supplementary Figures 7 and 8, available with the online version of this article).

Safety in escape population. The overall AE profile in patients who received escape therapy with TCZ-SC qw was comparable with that in patients who received TCZ-SC q2w continuously, and was similar between the escape-PBO and escape-TCZ-SC q2w groups (Table 1 and Table 2). There were 14 patients in the prior-PBO arm and 16 patients in the prior-TCZ-SC q2w arm who had 19 and 23 SAE, respectively; the most common were infections. A total of 7 patients

Table 2. Escape population: overview of cumulative AE and SAE from time of escape to Week $96^{\mathrm{a}}$. Values are rate/100 PY (95\% CI), unless otherwise specified.

\begin{tabular}{lcc}
\hline Variables & TCZ-SC q2w to TCZ-SC qw, $\mathrm{n}=98$ & PBO to TCZ-SC qw, $\mathrm{n}=91$ \\
\hline PY exposure & 126.54 & 129.27 \\
AE & $331.90(300.91-365.21)$ & $360.48(328.49-394.74)$ \\
$\quad$ No. events & 420 & 466 \\
SAE & $18.18(11.52-27.27)$ & $14.7(8.85-22.95)$ \\
$\quad$ No. events & 23 & 19 \\
Withdrawals because of AE & $6.32(2.73-12.46)$ & $5.41(2.18-11.16)$ \\
$\quad$ No. events & 8 & 7 \\
Infections & $101.15(84.39-120.27)$ & $95.92(79.78-114.37)$ \\
$\quad$ No. events & 128 & 124 \\
Serious infections & $9.48(4.90-16.56)$ & $5.41(2.18-11.16)$ \\
$\quad$ No. events & 12 & 7 \\
Injection-site reactions & $11.85(6.63-19.55)$ & $11.60(6.49-19.14)$ \\
$\quad$ No. events & 15 & 15 \\
Hypersensitivity events ${ }^{\mathrm{b}}$ & $3.16(0.86-8.09)$ & $6.19(2.67-12.19)$ \\
$\quad$ No. events & 4 & 8 \\
Serious hypersensitivity events ${ }^{\mathrm{c}}$ & 0 & 0 \\
Malignancies & $1.58(0.19-5.71)$ & $3.87(1.26-9.03)$ \\
$\quad$ No. events & 2 & 5 \\
Serious myocardial infarction & 0 & $1.55(0.19-5.59)$ \\
$\quad$ No. events & & 2 \\
Serious bleeding events & 0 & $0.77(0.02-4.31)$ \\
$\quad$ No. events & 0 & 1 \\
Deaths & & $0.77(0.02-4.31)$ \\
$\quad$ No. events & & 1 \\
\hline
\end{tabular}

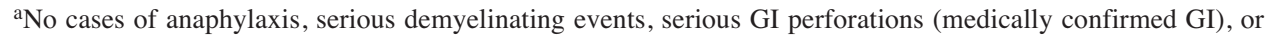
serious hepatic events were reported. ${ }^{b}$ Hypersensitivity events defined as all $\mathrm{AE}$ (except for injection-site reactions) that occurred during or within $24 \mathrm{~h}$ of injection and that were not judged "unrelated" to treatment by the investigator, regardless of whether they were consistent with hypersensitivity. ${ }^{c}$ Serious hypersensitivity events were defined as all hypersensitivity events that were reported as "serious" hypersensitivity. AE: adverse events; GI: gastrointestinal; PBO: placebo; PY: patient-years; qw: weekly; SAE: serious adverse events; TCZ-SC: subcutaneous tocilizumab. 
in the escape-PBO group and 8 in the escape-TCZ-SC q2w group developed 7 and 12 serious infections, respectively; the most common were cellulitis $(n=6)$ and pneumonia $(n=3)$. One patient in the escape-TCZ-SC group had an opportunistic infection of grade 2 atypical pneumonia, which resulted in interruption of study treatment; the event resolved without sequelae. The rates of $\mathrm{AE}, \mathrm{SAE}$, infections, serious infectious events, and withdrawals because of $\mathrm{AE}$ were broadly comparable over time, with wide and overlapping CI between the escape populations, and did not increase with continued exposure to TCZ-SC qw (Supplementary Figure 9, available with the online version of this article).

In the escape-PBO group, 5 malignancies occurred in 4 patients (Table 2): basal cell and squamous cell carcinoma occurred in 1 patient and leiomyosarcoma, malignant melanoma, and pancreatic carcinoma occurred in 1 patient each. In the escape-TCZ-SC group, 2 malignancies occurred in 2 patients: anal squamous cell carcinoma and breast cancer.

One death resulting from upper GI hemorrhage occurred in a patient from the escape-PBO group (Supplementary Table 3). Five escape patients ( 2 in the prior TCZ-SC arm and 3 in the prior $\mathrm{PBO}$ arm) had a hypersensitivity event. One patient in the escape-PBO group had 2 hypersensitivity events that led to study withdrawal (reported as grade 3 headache and grade 2 malaise). No cases of anaphylaxis, serious hypersensitivity events, serious demyelinating events, serious GI perforations (medically confirmed), or serious hepatic events were reported. In the escape-PBO group, 15 ISR occurred in 5 patients; most were injection-site erythema $(4.4 \%)$. One patient had a grade 3 ISR that led to dose modification from TCZ-SC qw to TCZ-SC q2w; the event resolved without sequelae. In the escape-TCZ-SC group, 15 ISR occurred in 6 patients. No ISR led to withdrawal.

Laboratory analysis of escape population. In the escapeTCZ-SC group, 2 patients (2.0\%) developed elevated (> $3 \times$ ULN) ALT; none had similarly elevated AST. The respective values in the escape-PBO group were 7 (7.7\%) and 5 (5.5\%). No patients met the Hy law criteria. One patient in the escape-TCZ group discontinued treatment because of grade 3 AE of elevated ALT and AST levels. Dose modification because of ALT elevation occurred in 5 patients $(5.1 \%)$ in the escape-TCZ group and in $7(7.7 \%)$ in the escape-PBO group, and because of elevated AST in $5(5.1 \%)$ and 4 patients $(4.4 \%)$, respectively.

Neutropenia was reported in $8.2 \%$ of escape-TCZ-SC patients and in $12.1 \%$ of escape-PBO patients. Thrombocytopenia was reported in 3 patients (3.1\%) in the escape-TCZ group and in 1 patient $(1.1 \%)$ in the escape-PBO group. One patient $(1.0 \%)$ in the escape-TCZ-SC group and $8(8.8 \%)$ in the escape-PBO group had shifts from normal to elevated total cholesterol $(\geq 240 \mathrm{mg} / \mathrm{dl})$. The incidence of shifts from normal $(<150 \mathrm{mg} / \mathrm{dl})$ to abnormal $(\geq 150$ to $<500 \mathrm{mg} / \mathrm{dl}$ ) triglycerides was slightly higher in the escape-PBO group [22 patients (24.2\%)] than in the escapeTCZ-SC group [17 patients (17.3\%)].
Immunogenicity in escape population. Three patients (3.3\%) in the escape-PBO group and 1 patient $(1.0 \%)$ in the escapeTCZ-SC q2w group developed anti-TCZ antibodies ${ }^{11}$. Two patients (2.2\%) in the escape-PBO group and 1 patient (1.0\%) in the escape-TCZ-SC q $2 \mathrm{w}$ group had a positive neutralizing antibody assay after the initiation of escape therapy. No patients who developed anti-TCZ antibodies had hypersensitivity events that led to study withdrawal, anaphylaxis, ISR, or loss of efficacy.

\section{DISCUSSION}

The BREVACTA study demonstrated that the efficacy and safety of TCZ-SC q2w were maintained over 96 weeks. Patients who were rerandomized from PBO to TCZ-SC (PBO-TCZ-SC group) had continued improvements in efficacy after Week 24, and by Week 36 the results were comparable with those in patients who received TCZ-SC continuously from baseline and sustained through 2 years. Patients who escaped from TCZ-SC q2w to TCZ-SC qw also had clinical improvement, but did not achieve as high a percentage of ACR responses. TCZ-SC q2w and qw were well tolerated, and the rate of $\mathrm{AE}$ and frequency of laboratory abnormalities did not increase with continued exposure. No new safety signals were observed.

With the exception of ISR, the safety profile of the TCZ-SC arm in our study was consistent with the known safety profile of TCZ-IV and was comparable with that observed in the longterm extension of the TCZ-SC trials ${ }^{8,9}$. The death rates were similar between the TCZ-SC and PBOTCZ-SC groups, with wide and overlapping CI (1.14/100 PY, 95\% CI 0.46-2.34 and 0.65/100 PY, 95\% CI 0.02-3.61). In the 96-week SUMMACTA study, the death rate/100 PY for patients receiving TCZ-SC $162 \mathrm{mg}$ qw was 0.39 (95\% CI 0.11-1.01), and was 0.49 (95\% CI, 0.13-1.25) for patients receiving TCZ-IV $8 \mathrm{mg} / \mathrm{kg}$ every 4 weeks $^{8}$. In a pooled analysis of TCZ-IV trials, the death rate for TCZ $8 \mathrm{mg} / \mathrm{kg}$ was $0.75 / 100 \mathrm{PY}$ and for the all-exposure TCZ population, it was $0.45 / 100 \mathrm{PY}^{12}$.

There was no intention to directly compare the TCZ-SC arm with the PBO-TCZ-SC arm; the data are presented for completeness. In the PBO-TCZ-SC arm, AE in patients who withdrew, died, or escaped in the first 24 weeks were not included, which makes it appear that this arm has fewer safety events. For patients who continuously received TCZ-SC in the double-blind and open-label periods, all events were included. Therefore, the event rates in the study arms contain data from different periods of the study and cannot be directly compared.

The percentage of patients with anti-TCZ antibodies remained low, comparable across the treatment arms and similar to those in other TCZ-SC studies ${ }^{8,9}$. No correlation between antibody development, hypersensitivity reactions, or loss of efficacy was observed.

Efficacy was similar between the TCZ-SC and PBO-

Personal non-commercial use only. The Journal of Rheumatology Copyright (C) 2018. All rights reserved. 
TCZ-SC arms among patients who weighed $<60 \mathrm{~kg}$, and among patients who weighed 60 to $<100 \mathrm{~kg}$. ACR response rates in the TCZ-SC group were lower in patients who weighed $\geq 100 \mathrm{~kg}$ at baseline than in the other weight subgroups. This finding should be interpreted cautiously because of the small number of patients who weighed $\geq 100$ $\mathrm{kg}$; it is consistent with observations from Week $24^{6}$. A similar trend was reported in TCZ-IV studies ${ }^{5}$, and in other studies, obese patients had lower responses to anti-TNF agents $^{13}$.

Efficacy responses of patients who escaped to TCZ-SC qw from PBO were comparable with those in the TCZ-SC $\mathrm{q} 2 \mathrm{w}$ arm. Patients who escaped from TCZ-SC $\mathrm{q} 2 \mathrm{w}$ to TCZ-SC qw also had improvements in ACR responses and DAS28 remission when receiving TCZ-SC qw. The lower but still improved response suggests that some patients who did not respond or only partially responded to TCZ-SC q2w may benefit from more frequent dosing.

In the escape population, $\mathrm{AE}$ remained consistent with the known safety profile of TCZ, and did not increase. The most frequent $\mathrm{AE}$ following escape were similar to the main population and included infections and infestations, GI disorders, and investigations. Although the rates of SAE, withdrawals for $\mathrm{AE}$, and infections were slightly higher in escape patients than those who received TCZ-SC q2w continuously, the rates were similar to those in the $\mathrm{PBO}$ arm during the double-blind period ${ }^{6}$, and the CI overlap.

TCZ-SC q2w demonstrated longterm efficacy, including sustained ACR responses, over 96 weeks. The safety profile of TCZ-SC at 96 weeks was comparable with that at 24 weeks. Improvement in efficacy was seen in some patients who did not initially respond to TCZ-SC q2 $\mathrm{w}$ and who then received TCZ-SC qw.

\section{ACKNOWLEDGMENT}

We thank all the investigators and patients who participated in the study, and all members of the BREVACTA (NA25220) study team. Support for third-party writing assistance for this manuscript, furnished by Denise Kenski, $\mathrm{PhD}$, of Health Interactions, was provided by F. Hoffmann-La Roche Ltd.

\section{ONLINE SUPPLEMENT}

Supplementary material accompanies the online version of this article.

\section{REFERENCES}

1. Emery P, Keystone E, Tony HP, Cantagrel A, van Vollenhoven R, Sanchez A, et al. IL-6 receptor inhibition with tocilizumab improves treatment outcomes in patients with rheumatoid arthritis refractory to anti-tumour necrosis factor biologicals: results from a 24-week multicentre randomised placebo-controlled trial. Ann Rheum Dis 2008;67:1516-23.
2. Genovese MC, McKay JD, Nasonov EL, Mysler EF, da Silva NA, Alecock E, et al. Interleukin-6 receptor inhibition with tocilizumab reduces disease activity in rheumatoid arthritis with inadequate response to disease-modifying antirheumatic drugs: the tocilizumab in combination with traditional disease-modifying antirheumatic drug therapy study. Arthritis Rheum 2008;58:2968-80.

3. Jones G, Sebba A, Gu J, Lowenstein MB, Calvo A, Gomez-Reino $\mathrm{JJ}$, et al. Comparison of tocilizumab monotherapy versus methotrexate monotherapy in patients with moderate to severe rheumatoid arthritis: the AMBITION study. Ann Rheum Dis 2010;69:88-96

4. Smolen JS, Beaulieu A, Rubbert-Roth A, Ramos-Remus C, Rovensky J, Alecock E, et al. Effect of interleukin-6 receptor inhibition with tocilizumab in patients with rheumatoid arthritis (OPTION study): a double-blind, placebo-controlled, randomised trial. Lancet 2008;371:987-97.

5. Burmester GR, Rubbert-Roth A, Cantagrel A, Hall S, Leszczynski P, Feldman D, et al. A randomised, double-blind, parallel-group study of the safety and efficacy of subcutaneous tocilizumab versus intravenous tocilizumab in combination with traditional disease-modifying antirheumatic drugs in patients with moderate to severe rheumatoid arthritis (SUMMACTA study). Ann Rheum Dis 2014;73:69-74.

6. Kivitz A, Olech E, Borofsky M, Zazueta BM, Navarro-Sarabia F, Radominski SC, et al. Subcutaneous tocilizumab versus placebo in combination with disease-modifying antirheumatic drugs in patients with rheumatoid arthritis. Arthritis Care Res 2014;66:1653-61.

7. Ogata A, Tanimura K, Sugimoto T, Inoue H, Urata Y, Matsubara T, et al. Phase III study of the efficacy and safety of subcutaneous versus intravenous tocilizumab monotherapy in patients with rheumatoid arthritis. Arthritis Care Res 2014;66:344-54.

8. Burmester GR, Rubbert-Roth A, Cantagrel A, Hall S, Leszczynski P, Feldman D, et al. Efficacy and safety of subcutaneous tocilizumab versus intravenous tocilizumab in combination with traditional DMARDs in patients with RA at week 97 (SUMMACTA). Ann Rheum Dis 2016;75:68-74.

9. Ogata A, Amano K, Dobashi H, Inoo M, Ishii T, Kasama T, et al. Longterm safety and efficacy of subcutaneous tocilizumab monotherapy: results from the 2-year open-label extension of the MUSASHI Study. J Rheumatol 2015;42:799-809.

10. van der Heijde D. How to read radiographs according to the Sharp/van der Heijde method. J Rheumatol 2000;27:261-263.

11. Stubenrauch K, Wessels U, Birnboeck H, Ramirez F, Jahreis A, Schleypen J. Subset analysis of patients experiencing clinical events of a potentially immunogenic nature in the pivotal clinical trials of tocilizumab for rheumatoid arthritis: Evaluation of an antidrug antibody ELISA using clinical adverse event-driven immunogenicity testing. Clin Ther 2010;32:1597-609.

12. Genovese MC, Rubbert-Roth A, Smolen JS, Kremer J, Khraishi M, Gomez-Reino J, et al. Longterm safety and efficacy of tocilizumab in patients with rheumatoid arthritis: a cumulative analysis of up to 4.6 years of exposure. J Rheumatol 2013;40:768-80.

13. Gremese E, Carletto A, Padovan M, Atzeni F, Raffeiner B, Giardina $\mathrm{AR}$, et al. Obesity and reduction of the response rate to anti-tumor necrosis factor alpha in rheumatoid arthritis: an approach to a personalized medicine. Arthritis Care Res 2013;65:94-100. 